Research Article

\title{
Potential Functional Variants in DNA Repair Genes Are Associated with Efficacy and Toxicity of Radiotherapy in Patients with Non-Small-Cell Lung Cancer
}

\author{
Zhiguang Yang and Zhaoyu Liu \\ Department of Radiology, Shengjing Hospital of China Medical University, Shenyang 110000, China \\ Correspondence should be addressed to Zhaoyu Liu; liuzy1226@126.com
}

Received 6 April 2020; Accepted 9 June 2020; Published 24 June 2020

Academic Editor: Vincenzo Coppola

Copyright (c) 2020 Zhiguang Yang and Zhaoyu Liu. This is an open access article distributed under the Creative Commons Attribution License, which permits unrestricted use, distribution, and reproduction in any medium, provided the original work is properly cited.

\begin{abstract}
Background. Lung cancer is one of the leading causes of cancer-related deaths. Radiotherapy, either alone or with chemotherapy, is still the primary treatment for patients with non-small-cell lung cancer (NSCLC). There are variations in how patients with NSCLC respond to radiotherapy and how toxic the therapy is. DNA repair gene polymorphisms are related to cancer development; however, their association with radiotherapy outcomes remains unknown. We hypothesized that gDNA repair gene variation could affect the efficacy and toxicity of radiotherapy in patients with NSCLC. Methods. A total of 486 histologically confirmed patients with NSCLC were recruited from the Shengjing Hospital of China Medical University from July 2015 to September 2019. Eleven potentially functional single nucleotide polymorphisms (SNPs) in four DNA repair genes (XRCC1, $X R C C 2, X P D$, and $M S H 2$ ) were genotyped in these patients. A multiple factor logistic regression analysis was used to assess the association between these SNPs and the efficacy and toxicity of radiotherapy. Results. Three SNPs, rs25487 (XRCC1), rs3218556 (XRCC2), and rs13181 (XPD), were all significantly associated with the efficacy of radiotherapy. The allele frequencies of the rs25487 CC genotype $(\mathrm{OR}=0.457,95 \% \mathrm{CI}=0.259-0.804, p=0.006)$ and the rs3218556 AG or AA genotypes (AG genotype: $\mathrm{OR}=0.664,95 \% \mathrm{CI}=0.442-0.999, p=0.049$; AA genotype: $\mathrm{OR}=0.380,95 \% \mathrm{CI}=0.181-0.795, p=0.008$ ) were both significantly higher in the response group than in the nonresponse group. For rs13181, the radiotherapy efficacy was associated with the heterozygous genotype GT $(\mathrm{OR}=1.663,95 \% \mathrm{CI}=1.057-2.614, p=0.027)$. Statistically significant associations between radiationinduced toxic reactions and rs25487 (XRCC1), rs3218556 (XRCC2), and rs13181 (XPD) were also observed. The rs13181GT genotype was associated with lower toxic reactions than the TT genotype $(\mathrm{OR}=1.680,95 \% \mathrm{CI}=1.035-2.728, p=0.035)$. Conclusions. The variants rs25487 (XRCC1), rs3218556 (XRCC2), and rs13181 (XPD) all contribute to the efficacy and toxicity of radiotherapy in patients with NSCLC. Our findings may clarify the predictive value of DNA repair genes for prognosis in patients with NSCLC after radiotherapy. Further investigation of more genes and samples should be performed to confirm our findings.
\end{abstract}

\section{Introduction}

Lung cancer accounts for a large proportion of cancer patients worldwide and is the leading cause of cancer-related deaths [1]. There were 9.6 million cancer-related deaths in $2018,18.4 \%$ of which were caused by lung cancer [2]. In China, about $28 \%$ and $23 \%$ of cancer-related death in 2012 occurred as a result of lung cancer in men and women, respectively. Non-small-cell lung cancer (NSCLC), including lung adenocarcinoma (LUAD) and lung squamous cell carcinoma (LUSC), accounts for approximately $85 \%$ of all lung cancers [3]. With a better understanding of tumor biology, there has been a breakthrough in the treatment of lung cancer. However, radiotherapy, either alone or with chemoradiotherapy, is still the primary treatment or palliative care for many patients, especially for locally advanced NSCLC patients who cannot be surgically resected. There is often a significant difference in treatment effects and toxic reactions in NSCLC patients undergoing standard radiotherapy $[4,5]$. Genetic variations between individuals or 
TABle 1: Clinical characteristics and demographics of the NSCLC patients.

\begin{tabular}{lc}
\hline Characteristic & NSCLC patients $(n=486)$ \\
\hline Age (years) & $62(33-84)$ \\
Gender (\%) & $328(67.5 \%)$ \\
$\quad$ Male & $158(32.5 \%)$ \\
Female & \\
Smoke history (\%) & $244(50.2 \%)$ \\
$\quad$ Smokers & $242(49.8 \%)$ \\
$\quad$ Never smokers & \\
Histology (\%) & $307(63.2 \%)$ \\
$\quad$ Squamous cell carcinoma & $179(36.8 \%)$ \\
$\quad$ Adenocarcinoma & \\
Family history of cancer (\%) & $76(15.7 \%)$ \\
$\quad$ Yes & $410(84.3 \%)$ \\
$\quad$ No & \\
Chemotherapy (\%) & $383(78.8 \%)$ \\
$\quad$ Yes & $103(21.2 \%)$ \\
$\quad$ No & \\
Response (\%) & $234(48.1 \%)$ \\
$\quad$ Response (CR + PR) & $252(51.9 \%)$ \\
$\quad$ Nonresponse (SD + PD)
\end{tabular}

tumors are the main reasons for the differences in sensitivity to radiotherapy [6].

Ionizing radiation leads to cell death mainly by creating double-strand breaks (DSB) [7] or through damage to cell membranes [8]. Studies have shown that DNA repair genes, such as ERCC1 [9], XRCC1 [10], and XPA [11], play a key role in the different repair processes that are invoked in cells following DNA damage. There are several DNA repair pathways in the human body, of which the nucleotide excision repair (NER), mismatch repair (MMR), and homologous recombination (HR) systems repair the damaged DNA after the formation of cross-link chains, whereas the base excision repair (BER) pathway repairs it before the formation of cross-link chains. XRCC1 and XRCC2 are involved in the BER and HR pathways, respectively, and have been associated with the occurrence of cancer $[12,13]$. MutS homolog 2 (MSH2), a key component of the MMR pathway, plays an important role in the development of neoplastic diseases [14]. The Xeroderma pigmentosum group $D(X P D)$ gene, another important DNA repair gene, has been reported to contribute to the risk of human cancer [15].

Although the relationship between polymorphisms in DNA repair genes and the development of cancer has been well explored in previous studies, few reports have investigated the interrelationship between gene polymorphisms and radiosensitivity or radiotherapy toxicity in patients with NSCLC. To explore the possible association between DNA repair gene variations and radiotherapy sensitivity and associated toxicity, we evaluated eleven single nucleotide polymorphisms (SNPs) in four DNA repair genes (XRCC1, $X R C C 2, X P D$, and $M S H 2$ ).

\section{Materials and Methods}

2.1. Study Population. In this study, a total of 486 histologically confirmed NSCLC patients were recruited from the
Shengjing Hospital of China Medical University from July 2015 to September 2019. The detailed characteristics of these patients are described in Table 1. The inclusion criteria were as follows: (1) patients who were diagnosed as having primary NSCLC and not eligible for surgery; (2) patients who underwent a lung biopsy and had a confirmed histopathological diagnosis of NSCLC; (3) patients who received a standard dose of radiotherapy; (4) patients who had no recurrent disease; (5) patients who had no other malignant tumors or history of radiotherapy. All participants or family members signed an informed consent form before blood collection and analysis. This study was approved by the ethics committee of the Shengjing Hospital of China Medical University.

2.2. Radiotherapy Treatment and Evaluation. All patients were treated with three-dimensional conformal radiation therapy (3D-CRT) or intensity modulated radiation therapy (IMRT), with a total radiation dose of 50-70 Gy. Followups were conducted on all patients three months after radiotherapy, and their response to treatment was assessed using computed omography (CT) according to the Response Evaluation Criteria in Solid Tumors (RECIST) guidelines. There were four categories of response defined: complete response (CR), partial response (PR), stable disease (SD), and progressive disease (PD) [16]. In the present study, CR and PR were grouped as "responders," whereas SD and PD were grouped as "nonresponders." Radiation-induced toxic reactions were graded according to the Radiation Therapy Oncology Group or European Organization for Research and Efficacy of Cancer (RTOG/EORTC) guidelines. Patients with grade 0 and grade 1 reactions were considered to have "low-toxic reactions" and those with grades $2-5$ reactions, "high-toxic reactions."

2.3. Selection of SNPs and Genotyping. Four DNA repair genes, XRCC1, XRCC2, MSH2, and XPD, which had previously been shown to be positively associated with the development of cancer, were selected for analysis. SNP genotypes were downloaded from the 1000 Genomes project (https://www.internationalgenome.org/) and analyzed using Haploview 4.2 software (https://www.broadinstitute.org/ haploview/haploview). Candidate SNPs which met the following criteria were included: (1) SNPs that had a minor allele frequency $(\mathrm{MAF})>0.1$ in Han Chinese in Beijing (CHB); (2) SNPs with a potential function, such as missense variations causing amino acid changes or were present in the $5^{\prime}$ or $3^{\prime}$ untranslated regions (UTRs) that could affect transcription factor binding site (TFBS) activity; (3) SNPs that have been reported in previous association studies. As a result of using these selection criteria, a total of 11 potentially functional SNPs in DNA repair genes were selected. These included 4 XRCC1 SNPs (rs25487 (exon 10), rs25489 (exon 9), rs1799782 (exon (6) and rs3213245 (5' UTR)), 3 XRCC2 SNPs (rs3218556 ( $3^{\prime}$ UTR), rs3218544 (3' UTR), rs3218385 (5' UTR)), 2 MSH2 SNPs (rs2303424 (exon 16) and rs2303425 (5' UTR)), and 2 XPD SNPs (rs13181 (exon 10) and rs238419 ( $3^{\prime}$ UTR)). 
TABLE 2: Association between candidate SNPs and efficacy of radiotherapy.

\begin{tabular}{|c|c|c|c|c|c|c|}
\hline Gene & SNPs & Genotype & $\mathrm{CR}+\mathrm{PR}$ & $\mathrm{SD}+\mathrm{PD}$ & OR $(95 \% \mathrm{CI})$ & $p$ \\
\hline \multirow{16}{*}{$X R C C 1$} & \multirow{4}{*}{ rs25487 } & CC & 130 & 111 & Reference & Reference \\
\hline & & $\mathrm{CT}$ & 81 & 98 & $0.706(0.479-1.041)$ & 0.078 \\
\hline & & TT & 23 & 43 & $0.457(0.259-0.804)$ & 0.006 \\
\hline & & $\mathrm{CT}+\mathrm{TT}$ & 104 & 141 & $0.630(0.440-0.901)$ & 0.011 \\
\hline & \multirow{4}{*}{ rs25489 } & CC & 190 & 203 & Reference & Reference \\
\hline & & CT & 42 & 47 & $0.955(0.602-1.514)$ & 0.844 \\
\hline & & $\mathrm{TT}$ & 2 & 2 & $1.068(0.149-7.661)$ & 0.947 \\
\hline & & $\mathrm{CT}+\mathrm{TT}$ & 44 & 49 & $0.959(0.610-1.509)$ & 0.858 \\
\hline & \multirow{4}{*}{ rs1799782 } & GG & 128 & 121 & Reference & Reference \\
\hline & & AG & 84 & 97 & $0.819(0.558-1.201)$ & 0.306 \\
\hline & & $\mathrm{AA}$ & 22 & 34 & $0.612(0.339-1.105)$ & 0.101 \\
\hline & & $\mathrm{AG}+\mathrm{AA}$ & 106 & 131 & $0.765(0.535-1.093)$ & 0.141 \\
\hline & \multirow{4}{*}{ rs3213245 } & AA & 182 & 181 & Reference & Reference \\
\hline & & AG & 53 & 66 & $0.799(0.527-1.211)$ & 0.289 \\
\hline & & GG & 3 & 5 & $0.597(0.141-2.534)$ & 0.479 \\
\hline & & $A G+G G$ & 56 & 71 & $0.784(0.523-1.178)$ & 0.241 \\
\hline \multirow{12}{*}{$X R C C 2$} & \multirow{4}{*}{ rs3218544 } & GG & 82 & 78 & Reference & Reference \\
\hline & & AG & 114 & 133 & $0.815(0.547-1.214)$ & 0.315 \\
\hline & & $\mathrm{AA}$ & 38 & 41 & $0.882(0.514-1.512)$ & 0.647 \\
\hline & & $\mathrm{AG}+\mathrm{AA}$ & 152 & 174 & $0.831(0.569-1.214)$ & 0.338 \\
\hline & \multirow{4}{*}{ rs3218385 } & AA & 172 & 172 & Reference & Reference \\
\hline & & $\mathrm{AC}$ & 53 & 73 & $0.726(0.481-1.096)$ & 0.127 \\
\hline & & CC & 9 & 7 & $1.286(0.468-3.530)$ & 0.625 \\
\hline & & $\mathrm{AC}+\mathrm{CC}$ & 62 & 80 & $0.775(0.523-1.148)$ & 0.203 \\
\hline & \multirow{4}{*}{ rs3218556 } & GG & 166 & 149 & Reference & Reference \\
\hline & & AG & 57 & 77 & $0.664(0.442-0.999)$ & 0.049 \\
\hline & & AA & 11 & 26 & $0.380(0.181-0.795)$ & 0.008 \\
\hline & & $\mathrm{AG}+\mathrm{AA}$ & 68 & 103 & $0.593(0.406-0.865)$ & 0.006 \\
\hline \multirow{8}{*}{$\mathrm{MSH} 2$} & \multirow{4}{*}{ rs2303424 } & GG & 86 & 102 & Reference & Reference \\
\hline & & AG & 117 & 129 & $1.076(0.735-1.574)$ & 0.707 \\
\hline & & AA & 31 & 20 & $1.599(0.868-2.945)$ & 0.131 \\
\hline & & $\mathrm{AG}+\mathrm{AA}$ & 148 & 149 & $1.178(0.817-1.699)$ & 0.380 \\
\hline & \multirow{4}{*}{ rs2303425 } & $\mathrm{TT}$ & 147 & 166 & Reference & Reference \\
\hline & & $\mathrm{CT}$ & 72 & 62 & $1.311(0.874-1.968)$ & 0.190 \\
\hline & & CC & 15 & 24 & $0.706(0.357-1.396)$ & 0.315 \\
\hline & & $\mathrm{CT}+\mathrm{CC}$ & 87 & 86 & $1.142(0.788-1.657)$ & 0.483 \\
\hline \multirow{8}{*}{$X P D$} & \multirow{4}{*}{ rs238419 } & CC & 79 & 95 & Reference & Reference \\
\hline & & $\mathrm{CT}$ & 101 & 110 & $1.104(0.738-1.651)$ & 0.629 \\
\hline & & $\mathrm{TT}$ & 54 & 47 & $1.382(0.845-2.259)$ & 0.197 \\
\hline & & $\mathrm{CT}+\mathrm{TT}$ & 155 & 157 & $1.187(0.818-1.722)$ & 0.366 \\
\hline & \multirow{4}{*}{ rs13181 } & TT & 176 & 209 & Reference & Reference \\
\hline & & GT & 56 & 40 & $1.663(1.057-2.614)$ & 0.027 \\
\hline & & GG & 2 & 3 & $0.792(0.131-4.791)$ & 0.799 \\
\hline & & $\mathrm{GT}+\mathrm{GG}$ & 58 & 43 & $1.602(1.029-2.493)$ & 0.036 \\
\hline
\end{tabular}

The $p$ values in bold represent a statistically significant association. All data are adjusted for age, gender, smoking history, cancer histology, family history, and treatment with chemotherapy.

Genomic DNA from all patients was extracted using a TIANamp Genomic DNA Kit (Tiangen Biotech, Beijing, China). Genotyping was performed using the TaqMan methodology and an Applied Biosystems 7500 FAST RealTime PCR System (Applied Biosystems, Foster City, CA, USA) according to the manufacturer's instructions. The predesigned SNP-genotyping assay mixture, containing the PCR primers and probes, were supplied by Applied Biosystems. The PCR amplification mix was prepared as follows: $25 \mu \mathrm{L}$ of master mix (Applied Biosystems), $10 \mu \mathrm{L}$ of DNA, and $15 \mu \mathrm{L}$ of $\mathrm{ddH}_{2} \mathrm{O}$. Amplification was performed under the following conditions: $50^{\circ} \mathrm{C}$ for $2 \mathrm{~min}, 95^{\circ} \mathrm{C}$ for $10 \mathrm{~min}, 40$ cycles of $95^{\circ} \mathrm{C}$ for $15 \mathrm{sec}, 60^{\circ} \mathrm{C}$ for $1 \mathrm{~min}$. Three negative controls (no DNA) and three positive controls in each 96well plate were used to ensure the accuracy of the PCR amplification. In addition, $10 \%$ of randomly repeated samples were used for quality control.

2.4. Statistical Analysis. Statistical analyses were performed using SPSS 22.0 software (SPSS, Chicago, IL, USA). The Hardy-Weinberg equilibrium was used to assess that all SNPs met the group representation. A multiple factor logistic regression was applied to assess the association 
between SNPs and efficacy of radiotherapy as well as radiation-induced toxicity reaction after adjusting for age, gender, smoking history, cancer histology, family history, and chemotherapy. A $p<0.05$ was considered statistically significant.

\section{Results}

3.1. Characteristics of Patients and Clinical Outcomes. The clinical characteristics and demographics of the 486 NSCLC patients are described in Table 1 . The proportion of male patients $(67.5 \%)$ was greater than double the proportion of female patients $(32.5 \%)$. The median age of all patients was 62 (ranging from 33 to 84). About half of the patients had a history of smoking. There were 307 (63.2\%) cases of squamous cell carcinoma and 179 (36.8\%) cases of adenocarcinoma. Seventy-six patients $(15.7 \%)$ had a family history of cancer and 383 (78.8\%) had been treated with chemotherapy. The response rate to radiotherapy was $48.1 \%$.

3.2. Associations between Candidate SNPs and Efficacy of Radiotherapy. The associations between candidate SNPs and the response to radiotherapy in NSCLS patients are shown in Table 2. All the candidate SNPs reached equilibrium according to the Hardy-Weinberg equilibrium test ( $p>0.05$, data not shown). The SNPs rs25487 (XRCC1), rs3218556 (XRCC2), and rs13181 (XPD) all showed a significant association with the efficacy of radiotherapy. The allele frequency of $\mathrm{rs} 25487 \mathrm{CC}$ genotype (OR $=0.457,95 \%$ $\mathrm{CI}=0.259-0.804, p=0.006)$ and the $\mathrm{rs} 3218556 \mathrm{AG}$ or AA genotypes $\quad(\mathrm{AG} \quad$ genotype: $\mathrm{OR}=0.664, \quad 95 \%$ $\mathrm{CI}=0.442-0.999, p=0.049 ; \mathrm{AA}$ genotype: $\mathrm{OR}=0.380,95 \%$ $\mathrm{CI}=0.181-0.795, p=0.008$ ) were significantly higher in the response group than in the nonresponse group. For rs25487, although the response rate between the CT and CC genotypes was not statistically significant $(p=0.078)$, a higher response rate was also observed when comparing the CT + CC genotype with the CC genotype, using a dominant model. For rs13181, a better radiotherapy efficacy was associated with the heterozygotic genotype GT $(\mathrm{OR}=1.663$, $95 \% \mathrm{CI}=1.057-2.614, p=0.027)$. There were no significant associations between the other SNPs and the efficacy of radiotherapy.

3.3. Associations between Candidate SNPs and RadiationInduced Toxic Reactions. The associations between candidate SNPs and radiation-induced toxic reactions are shown in Table 3. A statistically significant association between radiation-induced toxic reactions and rs25487 (XRCC1), rs3218556 (XRCC2), and rs13181 (XPD) was observed. For rs25487, the genotypes CT, TT, and CT + TT were associated with a severe toxic reaction compared to the CC genotype (all, $p<0.05$ ). For rs3218556, the ORs for cases with the AG, $\mathrm{AA}$, and AG + AA genotypes compared with homozygous CC genotype were $0.605(95 \% \mathrm{CI}=0.396-0.924, p=0.019)$, $0.279(95 \% \mathrm{CI}=0.116-0.675, p=0.003)$, and $0.540(95 \%$ $\mathrm{CI}=0.362-0.805, p=0.002)$, respectively. The rs13181GT genotype was associated with lower toxic reactions compared with the TT genotype $(\mathrm{OR}=1.680,95 \%$ $\mathrm{CI}=1.035-2.728, p=0.035)$.

\section{Discussion}

NSCLC is the leading cause of cancer-related death. Radiotherapy is an important treatment for NSCLC patients, especially for advanced NSCLC patients. However, there are significant differences in the efficacy of radiotherapy as well as in the incidence rate for radiation-induced toxic reactions. Identification of the key determinants that affect efficacy and toxicity is of paramount importance for the efficacy of radiotherapy in patients with NSCLC. Although numerous studies have reported that genetic polymorphisms in DNA repair genes are related to the development of cancer, their association with the outcomes of radiotherapy remains unknown. In the present study, we demonstrated that rs25487 (XRCC1), rs3218556 (XRCC2), and rs13181 (XPD) were associated with the efficacy and toxicity of radiotherapy in patients with NSCLC.

The potential functional variations of the four DNA repair genes were listed in Supplementary Table 1. The variations of rs25487 (c.1196A > G, p. Gln399Arg), rs25489 (c.839G > A, p. Arg280His), rs1799782 (c.580C > T, p. Arg194Trp) of XRCC1 gene, rs2303424 (c.2744A>G, p. Gln915Arg) of MSH2 gene, and rs13181 (c.2251A > C, p. Lys751Gln) of XPD gene cause amino acid changes and then affect the biological function of the protein. Also, other variations located in the $5^{\prime}$ or $3^{\prime}$ UTRs may affect the transcription factor binding site (TFBS) activity and then influence the DNA repair gene expression. All the variants may impact the clinical outcome of radiotherapy through these two ways.

Many genetic variants that are involved in DNA damage repair and the regulation of oxidative stress are associated with radiotherapy outcomes [17]. XRCC1, an important component of BER, has been reported to be associated with an increased risk of NSCLC in nonsmoking female patients with a history of exposure to cooking oil mist [10]. Genetic polymorphisms in XRCC1-194 and XRCC1-399 are also related to the risk of NSCLC [18]. Wang et al. [19] have reported that the presence of XRCC1 rs25489 had a significant impact on primary tumor efficacy at the end of radiotherapy and may act as a biomarker for the curative effect of radiotherapy. Zhai et al. [20] found that patients with nasopharyngeal carcinoma (NPC) carrying the XRCC1 codon $399 \mathrm{Gln} / \mathrm{Gln}$ genotype had a higher rate of tumor regression after radiotherapy. Another study showed that, in 114 patients with NPC, the XRCC1 rs25487 GA genotype was related with grade 3 dermatitis and grade 3 mucositis. In this study, a significant association was also observed between the rs 25489 CC genotype and a higher response rate to radiotherapy as well as lower toxic reaction. Although three other SNPs (rs25489, rs1799782, and rs3213245) in XRCC1 were also genotyped, no significant associations were observed. Due to the fact that XRCC1 rs25487 is clearly important in radiation sensitivity and the resultant toxic reactions, it could be considered as a biomarker that can be used to predict the clinical outcomes of radiotherapy. 
TABLE 3: Association between candidate SNPs and radiation-induced toxic reactions.

\begin{tabular}{|c|c|c|c|c|c|c|}
\hline Gene & SNPs & Genotype & Low toxic reactions & High toxic reactions & OR (95\% CI) & $p$ \\
\hline \multirow{16}{*}{ XRCC1 } & \multirow{4}{*}{ rs25487 } & CC & 188 & 74 & Reference & Reference \\
\hline & & $\mathrm{CT}$ & 114 & 69 & $0.650(0.435-0.972)$ & 0.035 \\
\hline & & $\mathrm{TT}$ & 23 & 18 & $0.503(0.257-0.986)$ & 0.043 \\
\hline & & $\mathrm{CT}+\mathrm{TT}$ & 137 & 87 & $0.620(0.424-0.907)$ & 0.013 \\
\hline & \multirow{4}{*}{ rs25489 } & $\mathrm{CC}$ & 257 & 122 & Reference & Reference \\
\hline & & $\mathrm{CT}$ & 65 & 38 & $0.812(0.515-1.279)$ & 0.369 \\
\hline & & $\mathrm{TT}$ & 3 & 1 & $0.424(0.147-13.83)$ & 0.759 \\
\hline & & $\mathrm{CT}+\mathrm{TT}$ & 68 & 39 & $0.828(0.528-1.297)$ & 0.409 \\
\hline & \multirow{4}{*}{ rs1799782 } & GG & 175 & 91 & Reference & Reference \\
\hline & & AG & 117 & 58 & $1.049(0.700-1.571)$ & 0.817 \\
\hline & & AA & 33 & 12 & $1.430(0.705-2.902)$ & 0.320 \\
\hline & & $\mathrm{AG}+\mathrm{AA}$ & 150 & 70 & $1.114(0.762-1.630)$ & 0.577 \\
\hline & \multirow{4}{*}{ rs3213245 } & AA & 248 & 117 & Reference & Reference \\
\hline & & AG & 74 & 43 & $0.812(0.525-1.255)$ & 0.348 \\
\hline & & GG & 3 & 1 & $1.415(0.146-13.75)$ & 0.764 \\
\hline & & $\mathrm{AG}+\mathrm{GG}$ & 77 & 44 & $0.826(0.537-1.270)$ & 0.383 \\
\hline \multirow{12}{*}{ XRCC2 } & \multirow{4}{*}{ rs3218544 } & GG & 117 & 49 & Reference & Reference \\
\hline & & AG & 162 & 83 & $0.817(0.534-1.251)$ & 0.353 \\
\hline & & AA & 46 & 28 & $0.688(0.387-1.224)$ & 0.202 \\
\hline & & $\mathrm{AG}+\mathrm{AA}$ & 208 & 111 & $0.785(0.523-1.177)$ & 0.241 \\
\hline & \multirow{4}{*}{ rs3218385 } & AA & 235 & 109 & Reference & Reference \\
\hline & & $\mathrm{AC}$ & 78 & 47 & $0.770(0.502-1.180)$ & 0.229 \\
\hline & & CC & 12 & 5 & $1.113(0.383-3.238)$ & 0.844 \\
\hline & & $\mathrm{AC}+\mathrm{CC}$ & 90 & 52 & $0.803(0.533-1.210)$ & 0.293 \\
\hline & \multirow{4}{*}{ rs3218556 } & GG & 238 & 96 & Reference & Reference \\
\hline & & AG & 78 & 52 & $0.605(0.396-0.924)$ & 0.019 \\
\hline & & AA & 9 & 13 & $0.279(0.116-0.675)$ & 0.003 \\
\hline & & $\mathrm{AG}+\mathrm{AA}$ & 87 & 65 & $0.540(0.362-0.805)$ & 0.002 \\
\hline \multirow{8}{*}{ MSH2 } & \multirow{5}{*}{ rs2303424 } & GG & 123 & 52 & Reference & Reference \\
\hline & & AG & 156 & 82 & $0.804(0.528-1.224)$ & 0.309 \\
\hline & & AA & 46 & 27 & $0.720(0.405-1.280)$ & 0.263 \\
\hline & & $\mathrm{AG}+\mathrm{AA}$ & 202 & 109 & $0.783(0.526-1.168)$ & 0.230 \\
\hline & & TT & 204 & 106 & Reference & Reference \\
\hline & \multirow{3}{*}{ rs2303425 } & $\mathrm{CT}$ & 104 & 45 & $1.201(0.788-1.830)$ & 0.394 \\
\hline & & CC & 17 & 10 & $0.883(0.391-1.997)$ & 0.765 \\
\hline & & $\mathrm{CT}+\mathrm{CC}$ & 121 & 55 & $1.143(0.769-1.698)$ & 0.508 \\
\hline \multirow{8}{*}{$X P D$} & \multirow{4}{*}{ rs238419 } & CC & 102 & 43 & Reference & Reference \\
\hline & & $\mathrm{CT}$ & 149 & 77 & $0.816(0.520-1.280)$ & 0.375 \\
\hline & & $\mathrm{TT}$ & 74 & 41 & $0.761(0.451-1.283)$ & 0.304 \\
\hline & & $\mathrm{CT}+\mathrm{TT}$ & 223 & 118 & $0.797(0.523-1.213)$ & 0.289 \\
\hline & \multirow{4}{*}{ rs13181 } & $\mathrm{TT}$ & 235 & 130 & Reference & Reference \\
\hline & & GT & 82 & 27 & $1.680(1.035-2.728)$ & 0.035 \\
\hline & & GG & 8 & 4 & $1.106(0.327-3.745)$ & 0.871 \\
\hline & & $\mathrm{GT}+\mathrm{GG}$ & 90 & 31 & $1.624(1.025-2.547)$ & 0.038 \\
\hline
\end{tabular}

The $p$ values in bold indicate a statistically significant association. All data are adjusted for age, gender, smoking history, cancer histology, family history, and treatment with chemotherapy.

Three SNPs (rs3218556, rs3218544, and rs3218385) in XRCC2, which is involved in the HR pathway, were also evaluated for a possible association with the efficacy and toxicity of radiotherapy in patients with NSCLC. Of these, only rs3218385 showed a significant association with both the efficacy and toxicity of radiotherapy. Popanda et al. [7] suggested that there was no relationship between the risk of acute skin toxicity and XRCC2 variations in patients with breast cancer receiving radiotherapy, which is inconsistent with our findings. Nevertheless, Yin et al. [21] genotyped six potentially functional SNPs in 228 patients with NSCLC who had been treated with definitive radiotherapy and found that
XRCC2 R188H SNPs was independent prognostic factor for overall survival. Qin et al. [22] also found that XRCC2deficient cancer cells were more sensitive to irradiation in vitro and speculated that the inhibition of XRCC2 expression or activity represents a potential therapeutic strategy for improving preoperative radiotherapy responses in patients with locally advanced rectal cancer. These results strongly suggest that XRCC2 plays an important role not only in the development of the cancer but also in radiotherapy outcomes.

MSH2 is an important MMR gene and several studies have suggested that variations in $\mathrm{MSH} 2$ are associated with 
sensitivity to radiotherapy and disease progression in rectal cancer patients $[23,24]$. However, the present study found that there was no association between two SNPs in this gene (rs2303424 and rs2303425) and the efficacy of radiotherapy. Xie et al. [25] also found that there was no correlation between survival time and $\mathrm{MSH} 2$ gene expression levels in patients with NSCLC who were treated with chemotherapy, which is consistent with our results. SNPs in the XPD gene have been widely studied and suggested to be genomic markers to predict the response to radiation dose and potentially guide personalized radiotherapy $[18,26]$. This study also discovered that $X P D$ rs13181 was associated with the progression and outcomes of patients with NSCLC after radiotherapy. Patients carrying the rs 13181 GT genotype who were treated with radiotherapy had a high level of toxic reaction coupled with low efficacy.

Despite the positive findings, there are also some limitations in this study. First, we were unable to explain how the DNA repair gene variations influenced the outcomes of radiotherapy. Secondly, too few SNPs were genotyped. We selected the potentially functional SNPs based on three criteria so that the SNPs did not cover all SNPs in the entire gene. Thirdly, our sample size is still too small. In this study, we failed to replicate the association between $\mathrm{MSH} 2$ and NSCLC patients. This could be due to the genetic heterogeneity in different population and a small number of samples included. Fourthly, gene-gene and gene-environment interactions were not analyzed. Additionally, some other important genes, such as ATM gene of DNA-damage sensing [27], ATG16L2 gene related to autophagy [28], and RUNX3 [29] gene in the methionine metabolic pathway that have been reported to be associated with the outcomes of NSCLS, were not included in this study.

In conclusion, we have identified patients with NSCLC carrying the rs25487 (XRCC1), rs3218556 (XRCC2), and rs13181 (XPD) SNPs, which appear to contribute to the efficacy and toxicity of radiotherapy. Our findings may be helpful in understanding the predictive value of examining DNA repair genes in the prognosis of patients with NSCLC after radiotherapy. Further investigation of more genes and samples should be carried out to confirm our findings. In addition, more studies should be implemented to explain the underlying molecular mechanisms to explain how these polymorphisms affect the response to radiotherapy and prospective clinical trials in patients with NSCLC.

\section{Data Availability}

The statistical data used to support the findings of this study are included within the article.

\section{Conflicts of Interest}

The authors declare that they have no conflicts of interest.

\section{Acknowledgments}

The study was supported by the National Natural Science Foundation of China (Grant nos. 81871465 and 81470086) and 345 Talent Project.

\section{Supplementary Materials}

Supplementary Table 1: genomic function of the ten SNPs in XRCC1, XRCC2, MSH2, and XPD genes. (Supplementary Materials)

\section{References}

[1] L. A. Torre, F. Bray, R. L. Siegel, J. Ferlay, J. Lortet-Tieulent, and A. Jemal, "Global cancer statistics," CA: A Cancer Journal for Clinicians, vol. 65, no. 2, pp. 87-108, 2012.

[2] F. Bray, J. Ferlay, I. Soerjomataram, R. L. Siegel, L. A. Torre, and A. Jemal, "Global cancer statistics 2018: GLOBOCAN estimates of incidence and mortality worldwide for 36 cancers in 185 countries," CA: A Cancer Journal for Clinicians, vol. 68, no. 6, pp. 394-424, 2018.

[3] J. R. Molina, P. Yang, S. D. Cassivi, S. E. Schild, and A. A. Adjei, "Non-small cell lung cancer: epidemiology, risk factors, treatment, and survivorship," Mayo Clinic Proceedings, vol. 83, no. 5, pp. 584-594, 2008.

[4] J. D. Bradley, R. Paulus, R. Komaki et al., "Standard-dose versus high-dose conformal radiotherapy with concurrent and consolidation carboplatin plus paclitaxel with or without cetuximab for patients with stage IIIA or IIIB non-small-cell lung cancer (RTOG 0617): a randomised, two-by-two factorial phase 3 study," The Lancet Oncology, vol. 16, no. 2, pp. 187-199, 2015.

[5] M. Kavadi, K. Bae, B. Movsas et al., "Higher biologically effective dose of radiotherapy is associated with improved outcomes for locally advanced non-small cell lung carcinoma treated with chemoradiation: an analysis of the radiation therapy oncology group," International Journal of Radiation Oncology Biology Physics, vol. 82, no. 1, pp. 425-434, 2012.

[6] C. Wang, H. Nie, Y. Li et al., "The study of the relation of DNA repair pathway genes SNPs and the sensitivity to radiotherapy and chemotherapy of NSCLC," Scientific Reports, vol. 6, Article ID 26526, 2016.

[7] O. Popanda, X. L. Tan, C. B. Ambrosone et al., "Genetic polymorphisms in the DNA double-strand break repair genes XRCC3, XRCC2, and NBS1 are not associated with acute side effects of radiotherapy in breast cancer patients," Cancer Epidemiology Biomarkers \& Prevention, vol. 15, no. 5, pp. 1048-1050, 2006.

[8] T.-L. Wang, Y.-W. Ren, H.-T. Wang, H. Yu, and Y.-X. Zhao, "Association of topoisomerase II (TOP2A) and dual-specificity phosphatase 6 (DUSP6) single nucleotide polymorphisms with radiation treatment response and prognosis of lung cancer in han Chinese," Medical Science Monitor, vol. 23, pp. 984-993, 2017.

[9] T.-P. Xu, H. Shen, L.-X. Liu, and Y.-Q. Shu, "Association of ERCC1-C118T and-C8092A polymorphisms with lung cancer risk and survival of advanced-stage non-small cell lung cancer patients receiving platinum-based chemotherapy: a pooled analysis based on 39 reports," Gene, vol. 526, no. 2, pp. 265-274, 2013.

[10] L. Wang, L.-L. Wang, D. Shang et al., "Gene polymorphism of DNA repair gene X-ray repair cross complementing group 1 and xeroderma pigmentosum group $\mathrm{D}$ and environment interaction in non-small-cell lung cancer for Chinese nonsmoking female patients," The Kaohsiung Journal of Medical Sciences, vol. 35, no. 1, pp. 39-48, 2019.

[11] I. Sullivan, J. Salazar, M. Majem et al., "Pharmacogenetics of the DNA repair pathways in advanced non-small cell lung 
cancer patients treated with platinum-based chemotherapy," Cancer Letters, vol. 353, no. 2, pp. 160-166, 2014.

[12] Z. Hu, H. Ma, F. Chen, Q. Wei, and H. Shen, "XRCC1 polymorphisms and cancer risk: a meta-analysis of 38 casecontrol studies," Cancer Epidemiology Biomarkers \& Prevention, vol. 14, no. 7, pp. 1810-1818, 2005.

[13] E. Eskandari, A. Rezaifar, and M. Hashemi, "XPG Asp1104His, XRCC2 Rs3218536 A/G and RAD51 135G/C gene polymorphisms and colorectal cancer risk: a metaanalysis," Asian Pacific Journal of Cancer Prevention: APJCP, vol. 18, no. 7, pp. 1805-1813, 2017.

[14] F. Hu, D. Li, Y. Wang et al., "Novel DNA variants and mutation frequencies of hMLH1 and hMSH2 genes in colorectal cancer in the Northeast China population," PLoS One, vol. 8, no. 4, Article ID e60233, 2013.

[15] A. Loghin, C. Bănescu, A. Nechifor-Boila et al., "XRCC3 Thr241Met and XPD Lys751Gln gene polymorphisms and risk of clear cell renal cell carcinoma," Cancer Biomarkers, vol. 16, no. 2, pp. 211-217, 2016.

[16] E. A. Eisenhauer, P. Therasse, J. Bogaerts et al., "New response evaluation criteria in solid tumours: revised RECIST guideline (version 1.1)," European Journal of Cancer, vol. 45, no. 2, pp. 228-247, 2009.

[17] Y. Rubinstein, B. Liu, J. Li et al., "Genetic variants in PI3K/ AKT pathway are associated with severe radiation pneumonitis in lung cancer patients treated with radiation therapy," Cancer Medicine, vol. 5, no. 1, pp. 24-32, 2016.

[18] Y. Du, Y. He, Z. Mei, L. Qian, J. Shi, and Z. Jie, “Association between genetic polymorphisms in XPD and XRCC1 genes and risks of non-small cell lung cancer in East Chinese Han population," The Clinical Respiratory Journal, vol. 10, no. 3, pp. 311-317, 2016.

[19] J. Wang, C. Guo, X. Gong et al., "The impacts of genetic polymorphisms in genes of base excision repair pathway on the efficacy and acute toxicities of (chemo) radiotherapy in patients with nasopharyngeal carcinoma," Oncotarget, vol. 8, no. 45, pp. 78633-78641, 2017.

[20] X.-M. Zhai, Q.-C. Hu, K. Gu, J.-P. Wang, J.-N. Zhang, and Y.-W. Wu, "Significance of XRCC1 Codon399 polymorphisms in Chinese patients with locally advanced nasopharyngeal carcinoma treated with radiation therapy," AsiaPacific Journal of Clinical Oncology, vol. 12, no. 1, pp. e125e132, 2016.

[21] M. Yin, Z. Liao, Y. J. Huang et al., "Polymorphisms of homologous recombination genes and clinical outcomes of nonsmall cell lung cancer patients treated with definitive radiotherapy," PLoS One, vol. 6, no. 5, Article ID e20055, 2011.

[22] C.-J. Qin, X.-M. Song, Z.-H. Chen et al., "XRCC2 as a predictive biomarker for radioresistance in locally advanced rectal cancer patients undergoing preoperative radiotherapy," Oncotarget, vol. 6, no. 31, pp. 32193-32204, 2015.

[23] J. Yang, X. Wang, S. M. Zou et al., "Genetic variations in MLH3 and MSH2 genes are associated with the sensitivity and prognosis in locally advanced rectal cancer patients receiving preoperative chemoradiotherapy," Zhonghua Zhong Liu Za Zhi [Chinese Journal of Oncology], vol. 40, no. 6, pp. 433-440, 2018.

[24] J. Yang, Y. Huang, Y. Feng et al., "Associations of genetic variations in mismatch repair genes MSH3 and PMS1 with acute adverse events and survival in patients with rectal cancer receiving postoperative chemoradiotherapy," Cancer Research and Treatment, vol. 51, no. 3, pp. 1198-1206, 2019.

[25] K.-J. Song, H.-E. He, A.-J. Sun, X.-B. Liu, L.-P. Sun, and X.-J. Dong, "Expression of ERCC1, MSH2 and PARP1 in non- small cell lung cancer and prognostic value in patients treated with platinum-based chemotherapy," Asian Pacific Journal of Cancer Prevention, vol. 15, no. 6, pp. 2591-2596, 2014.

[26] J.-Y. Jin, W. Wang, R. K. Ten Haken et al., "Use a survival model to correlate single-nucleotide polymorphisms of DNA repair genes with radiation dose-response in patients with non-small cell lung cancer," Radiotherapy and Oncology, vol. 117, no. 1, pp. 77-82, 2015.

[27] H. Xiong, Z. Liao, Z. Liu et al., "ATM polymorphisms predict severe radiation pneumonitis in patients with non-small cell lung cancer treated with definitive radiation therapy," International Journal of Radiation Oncology Biology Physics, vol. 85, no. 4, pp. 1066-1073, 2013.

[28] J. Wen, H. Liu, L. Wang et al., "Potentially functional variants of ATG16L2 predict radiation pneumonitis and outcomes in patients with non-small cell lung cancer after definitive radiotherapy," Journal of Thoracic Oncology, vol. 13, no. 5, pp. 660-675, 2018.

[29] K. Chen, H. Liu, Z. Liu et al., "Genetic variants in RUNX3, AMD1 and MSRA in the methionine metabolic pathway and survival in nonsmall cell lung cancer patients," International Journal of Cancer, vol. 145, no. 3, pp. 621-631, 2019. 